\title{
El nexo seguridad-desarrollo y la resiliencia como sucedáneos de la seguridad humana en las políticas de la Unión Europea: el caso del Sahel
}

\author{
Pablo Moral Martín*
}

\begin{abstract}
RESUMEN
Un cuarto de siglo después de la aparición del concepto, la seguridad humana apenas conserva un peso marginal en el acervo estratégico de la UE. La utilidad de sus preceptos originales ha quedado limitada a servir de orientación para la adopción de paradigmas sucedáneos, como son los casos del nexo seguridad-desarrollo y la resiliencia. En este artículo nos proponemos estudiar las diferencias entre estos conceptos y cómo ha evolucionado su relevancia en los documentos oficiales y estrategias de la Unión Europea (UE). Comprobamos cómo tanto el nexo seguridad-desarrollo como la resiliencia han prevalecido por resultar más prácticos y asumibles para la UE. En ellos, Bruselas ha encontrado la justificación teórica de las intervenciones en países cuyo "subdesarrollo" es percibido como una amenaza para la seguridad europea. Un ejemplo paradigmático de ello lo encontramos en el Sahel. En esta región se han llevado a cabo múltiples iniciativas bajo estas máximas, lo que ha resultado en una priorización de las cuestiones de seguridad sobre las de desarrollo y en el consecuente trasvase de recursos predestinados al desarrollo hacia asuntos de seguridad.
\end{abstract}

\section{Palabras clave}

seguridad humana; nexo seguridad-desarrollo; resiliencia; Unión Europea; Sahel.
* Pablo MORAL MARTÍN,

Doctorando en Ciencias Políticas y Jurídicas en la Universidad Pablo de Olavide de Sevilla. Graduado en RRIl y Máster en Estudios Euromediterráneos por la Universidad Complutense de Madrid.

Recibido: 30/09/2019 Aceptado: $18 / 12 / 2019$

\section{TitLE}

The security-development nexus and resilience as substitutes for human security in the policies of the European Union: the case of the Sahel

\begin{abstract}
Twenty-five years after the appearance of the concept, human security barely maintains a marginal weight in the EU's strategic acquis. The utility of its original precepts has been limited to serve as an orientation for the adoption of substitute paradigms, such as the security-development nexus and resilience. In this article we aim at studying the differences among these concepts and how their importance in official documents and strategies has developed. We check how the security-development nexus as well as the resilience have prevailed by becoming more practical and acceptable for the EU. In them it has found the theoretical justification for interventions in countries whose "underdevelopment" has been perceived as a threat to European security. A paradigmatic example of this can be found in the Sahel. In this region multiple initiatives following these concepts have been carried out, which has eventually entailed a prioritization of security issues over development ones and the transfer of resources predestined to development towards security matters.
\end{abstract}

\section{KEYWORDS}

Human security; security-development nexus; resilience; European Union; Sahel.

\section{DOI:}

https://doi.org// 0.15366/relacionesinternacionales2020.43.004

Formato de citación recomendado:

MORAL MARTÍN, Pablo, "El nexo seguridad-desarrollo y la resiliencia como sucedáneos de la seguridad humana en las políticas de la Unión Europea: el caso del Sahel”, en Relaciones Internacionales, n 43, 2020, pp. 69-86. 


\section{$\mathrm{I}$}

\section{ntroducción}

Tras veinticinco años de existencia, el prestigio e influencia de la seguridad humana en las agendas políticas internacionales se ha desvanecido gradualmente' .Tras gozar de popularidad en sus primeros lustros, el concepto ha envejecido y con él su potencial normativo: cada vez resulta más infrecuente aludir a la seguridad humana para justificar políticas o intervenciones en el exterior. No obstante, simultáneamente a la decadencia del uso de este concepto, han surgido otros paradigmas que recogen, en mayor o menor medida, parte de sus preceptos atribuidos. Es por ello que, si bien la seguridad humana no ha llegado a su primer cuarto de siglo de vida con la vigorosidad que otrora atesoró, su decadente recorrido existencial ha derivado en otros enfoques que se alejan de una visión clásica de la seguridad e inciden en la interdependencia entre esta y el desarrollo: el nexo seguridad-desarrollo y la resiliencia.

La evolución de la Política Exterior y de Seguridad Común de la UE (PESC) es un buen ejemplo de los claroscuros que ha tenido la aplicación práctica del concepto de la seguridad humana. La Unión, en general, ha sido muy timorata a la hora de abogar por la seguridad humana en sus documentos estratégicos, si bien una interpretación más complaciente podría esgrimir que sus postulados han servido de inspiración para paradigmas ulteriores. A pesar de que no ha sido frecuente desde Bruselas referirse explícitamente a la seguridad humana como base normativa de sus políticas, la reiterada alusión tanto al nexo seguridad-desarrollo como posteriormente a la resiliencia puede considerarse uno de los legados vigentes de la reconceptualización de la seguridad acaecida tras la Guerra Fría, que propició la irrupción del concepto de la seguridad humana.

En este artículo nos hemos propuesto evaluar la evolución del concepto de la seguridad humana en el acervo estratégico de la Unión Europea, tanto en significado como en relevancia. Del mismo modo, es nuestro objetivo comprobar hasta qué punto este paradigma ha sido efectivamente suplantado por otros sucedáneos. Para ello, en primer lugar, a modo de antecedentes teóricos y apoyándonos en autores prominentes, se abordará la convergencia entre la seguridad y el desarrollo y la resignificación de ambos conceptos en el escenario internacional. Posteriormente, estudiaremos la relevancia de la seguridad humana como herramienta estratégica para la elaboración de políticas por parte de la Unión Europea, mediante un análisis de los principales documentos desde que en 2003 se aprobara la Estrategia Europea de Seguridad.Y, en tercer lugar, en aras de dotar a este artículo de una mayor solidez empírica, estudiaremos el caso de las políticas de la UE hacia el Sahel, poniendo el foco a sus estrategias, objetivos y recursos destinados. Esta región, que para la UE engloba a 5 países — Burkina Faso, Chad, Malí, Mauritania y Níger- ${ }^{2}$ se ha convertido en un laboratorio de la política de seguridad y desarrollo de la UE en el exterior ${ }^{3}$ al incluir dos misiones civiles, en Níger y Malí, y también una militar en este último. La Estrategia de la UE para el Sahel no solo fue la primera impulsada por el Servicio Europeo de Acción Exterior (SEAE), creado en 201 I, para una región en concreto, sino que fue la primera que

DUFFIELD, M.,“Human Security and the Development-Security Nexus.An historical overview”, en Region pratica, 48, junio, 20 I 7, pp.6I-75.

2 CONSEJO DE LA UE, Council conclusions on implementation of the EU Strategy for Security and Development in the Sahel. FA Council Meeting Bruselas, I7/03/20I4.

3 VENTURI, Bernardo, The EU and the Sahel: A Laboratory of Experimentation for the Security-Migration-Development Nexus, Instituto Affari Internazionali, working paper 17/38, diciembre, 2019 
llevó por título "Estrategia para la Seguridad y el Desarrollo"4. Con el significativo aumento de la conflictividad en los años sucesivos, el Sahel ha pasado a ser considerado por Bruselas como "una amenaza endémica"' para la Unión, "la frontera geopolítica más meridional"6 de esta y "crucial"7 para su seguridad.Por todo ello, el estudio de este caso nos permitirá comprobar si las tendencias establecidas en cuanto al uso y desuso de los sucesivos conceptos en la estrategia general de la UE en materia de seguridad se materializan en esta región específica, poniendo especial énfasis en el grado de utilidad concedido a la seguridad humana y en las razones subyacentes que han influido en los cambios.

\section{La convergencia entre la seguridad y el desarrollo}

El concepto de la seguridad humana fue introducido en un informe del PNUD en 1994, si bien su irrupción no fue accidental ni repentina. Ya en los setenta y en los ochenta ${ }^{8}$ en el ámbito académico comenzaron a surgir voces críticas con los paradigmas imperantes de seguridad internacional, por su carácter estatocéntrico y militar?. Según Mark Duffield, a partir de los ochenta, con la expansión del neoliberalismo y el declive de las competencias del estado-nación, la concepción del desarrollo y los paradigmas de seguridad comenzaron a cambiar radicalmente ${ }^{10}$. De este modo, en el Norte Global se comenzó a percibir que la mayor interconexión del sistema mundial había magnificado la internacionalización de la inestabilidad en el Sur. Los flujos de refugiados, la capacidad para obstaculizar las relaciones comerciales y la expansión de redes relacionadas con el terrorismo y otras actividades criminales podían tener un mayor impacto en el Norte". El "subdesarrollo" pasó a considerarse un peligro, por lo que el fomento del desarrollo se convirtió en sinónimo de la búsqueda de la seguridad y al mismo tiempo, la seguridad se convirtió en un prerrequisito para conseguir el desarrollo sostenible.

Esta reconceptualización de la seguridad y el desarrollo propició, en los últimos años de los ochenta y la primera mitad de los noventa, la ampliación del concepto de seguridad con versiones más integrales y multifacéticas que atendían a elementos políticos, económicos, sociales y medioambientales, superando la concepción unidimensional de la seguridad nacional y las limitaciones de la seguridad colectiva ${ }^{12}$. Ejemplo de ello son las nociones de seguridad cooperativa ${ }^{13}$, seguridad global ${ }^{14}$ o seguridad democrática ${ }^{15}$; y en la misma línea podemos añadir la aparición del

\footnotetext{
4 SERVICIO EUROPEO DE ACCIÓN EXTERIOR (SEAE), Estrategia Global para la Política Exterior y de Seguridad de la Unión Europea, 20 I6.

5 PARLAMENTO EUROPEO, A Coherent EU Strategy for the Sahel, DG External Policies, Bruselas, 20I2, p.20.

6 Ibídem, p.5.

CONSEJO DE LA UE, EU Sahel Strategy. Regional Action Plan 20 I 5-2020. Bruselas, p.4.

8 SANHAUJA, José. y SCHUNEMANN, Julia., "El nexo seguridad-desarrollo: entre la construcción de la paz y la securitización de la ayuda" en SANHAUJA, J. (coord.): Construcción de la paz, seguridad y desarrollo.Visiones, políticas y actores, ICEI, Madrid, 2012.

9 PÉREZ DE ARMIÑO, Karlos, “El concepto y el uso de la seguridad humana: análisis crítico de sus potencialidades y riesgos”, en Revista CIDOB d'afers internacionals, N. 76, 2006, pp.59-77.

10 DUFFIELD, Mark, Las nuevas guerras en el mundo global. La convergencia entre desarrollo y seguridad, Catarata, Madrid, 200 I.

"Ibídem, p. 68

12 SANHAUJA, J, et. al., "El nexo seguridad....” op.cit. p.25.

13 La cooperación entre los estados para resolver problemas y amenazas comunes, particularmente aquellos de carácter no estatal. En MIHALKA, Michael, “Cooperative Security in the 2 I stCentury”, en Connections Vol. 4, No. 4 , 20 I5, pp. II 3-I22

14 "Una condición en la que la humanidad posee un patrón estable de estructuras y procesos, con instituciones, actitudes y comportamientos asociados, que trabaja hacia la reducción y la eliminación de amenazas de alcance global”. En KALDOR, Mary, y RANGELOR, lavor, The Handbook of Global Security Policy, John Wiley \& Sons, 2014.

15 Este término fue adoptado por el Consejo de Europa tras el fin de la Guerra Fría y con él se procuraba la seguridad de las democracias
} 
concepto de estados frágiles ${ }^{16} \circ$ fallidos ${ }^{17}$. Este surgimiento de nuevos paradigmas fue propiciado por las grandes transformaciones acontecidas en el contexto internacional. El fin de la Guerra Fría había hecho disminuir el riesgo de enfrentamiento nuclear entre las dos grandes potencias, del mismo modo que cada vez se hacían más infrecuentes los conflictos convencionales entre distintos estados ${ }^{18}$. En los ochenta y noventa los conflictos más usuales fueron conflictos civiles intraestatales producidos en contextos de crisis humanitarias ${ }^{19}$, por lo que se puso de manifiesto que, como argumenta Pérez de Armiño:

"Las amenazas eran más internas que externas, se derivaban en gran medida de factores socioeconómicos más que militares, afectaban a personas vulnerables más que al estado y este era muchas veces fuente de inseguridad y violencia" ${ }^{20}$.

Entre todos los conceptos surgidos entonces, el más relevante acabó siendo, en primera instancia, la seguridad humana, que comenzó a ocupar un papel central en el mundo académico y en la política exterior de países y organizaciones internacionales ${ }^{21}$. Su principal innovación era la modificación del enfoque estatocéntrico de la seguridad tradicional para situarlo en el individuo. Estar libres frente al temor y libres frente a las necesidades eran las máximas de esta nueva perspectiva, que concebía la seguridad desde una óptica multifacética, al abarcar siete ámbitos: seguridad económica, alimentaria, sanitaria, ambiental, personal, comunal y política ${ }^{22}$.

Sin embargo, el concepto partía de inicio con una serie de desventajas: su carácter amplio y multidimensional lo hacía ambiguo, vago y operativamente dificultoso. Es por ello que a la hora de llevarlo a la práctica, frecuentemente los decisores políticos lo han acotado de acuerdo a sus intereses. Fruto de esta limitación, desde los primeros años de vigencia de la seguridad humana se abrieron dos tendencias respecto a su aplicación: la versión amplia, correspondiente en mayor medida con la totalidad de sus preceptos; y una versión restringida que se centra en la protección frente al temor, habitualmente asociado a la violencia y el conflicto ${ }^{23}$. Dentro del enfoque amplio, Pérez de Armiño ${ }^{24}$ considera que existen dos dimensiones. Una dimensión cuantitativa, enfocada

liberales ante el "nacionalismo agresivo" o las ideologías "intolerantes y totalitarias". En CONSEJO DE EUROPA, Declaration of the Council of Europe's First Summit, Viena, octubre de 1993. Disponible en: http://www.cvce.eu/obj/declaration_of_the_council_of_europe_s_first_ summit_vienna_9_october_1993-end7c530b5-a7c9-43f9-95af-c28b3c8b50d3.html. Última consulta el 20/I I/2019

16 Los estados frágiles eran aquellos que no lograban ejercer la soberanía jurídica y empírica, es decir, la habilidad de controlar los medios de la violencia dentro de un territorio y de ejercitar una autoridad legítima con el fin de proveer un cierto grado de bienestar a sus ciudadanos. En SPEAR, Joanna,WILLIAMS, Paul, Security and development in global politics.A critical comparison, Georgetown University Press, Washington, D.C., 2012 p: 19

17 Según HELMAN, Gerald y RATNER, Steven, en "Saving Failed States" en Foreign Policy No. 89, pp. 3-20, 1992, eran aquellos estados que eran incapaces de funcionar como una entidad independiente y se caracterizaban por los conflictos civiles, el colapso del Gobierno y la privación económica. Otras definiciones posteriores incluyen los estados profundamente conflictivos, con fracturas sociales y étnicas, donde el Gobierno no puede garantizar la seguridad ni tampoco controlar las fronteras (ROTBERG, Robert, "Failed States, Collapsed States, Weak States: Causes and Indicators" en When States Fail: Causes and Consequences, Princeton, NJ: Princeton University Press, 2004).

18 ALBRECHT, Ulrich, et. al,. A human security doctrine for Europe: the Barcelona Report of the Study Group on Europe's Security Capabilities, Study Group on Europe's Security Capabilities, Barcelona, 2004

19 CHRISTIE, Ryerson., “Critical Voices and Human Security: to Endure, to Engage or to Critique?”, en Security Dialogue, n. ${ }^{\circ} 4 \mathrm{I}, 2010$. Entre los $^{\circ}$ ejemplos más paradigmáticos de ello destacan las guerras civiles en Etiopía (I974-199I) o en Somalia (desde I99I).

20 PÉREZ DE ARMIÑO, K., ¿Más allá de la seguridad humana? desafios y aportes de los estudios críticos de seguridad en Cursos de derecho internacional y relaciones internacionales de Vitoria-Gasteiz. N.I, 20I2, págs. 235-308.

2I SPEAR, J.,WILLIAMS, P., Security and op. cit. p.I3.

22 PNUD, Human Development Report 1994, New York, Oxford, Oxford University Press, 1994.

${ }^{23}$ NEWMAN, Edward, “Critical Human Security Studies”, en Review of International Studies, n.36, 20 I0, p. 80.

24 PÉREZ DE ARMIÑO, K., El concepto y el uso..., op.cit. 
en la provisión de las necesidades materiales para garantizar la subsistencia —alimentos, salud, vivienda o educación-; y una dimensión cualitativa, de carácter político, que recoge aspectos vinculados a la dignidad humana como la participación en los asuntos de la comunidad o la autonomía personal. Por su parte, el enfoque restringido fue el que acabó imponiéndose con más frecuencia en las agendas internacionales, en ocasiones reducido a una versión minimalista que asociaba la seguridad humana con la amenaza extrema del genocidio y los crímenes de lesa humanidad ${ }^{25}$.

Además de sus limitaciones a la hora de llevarlo a la práctica, a la progresiva merma de la popularidad del concepto contribuyó, por un lado, que sirviese de justificación teórica del creciente intervencionismo de Naciones Unidas en países del Sur en la década de los noventa ${ }^{26}$; y por otro, el advenimiento de la guerra global contra el terror preconizada por Estados Unidos tras los ataques del II de septiembre en Nueva York. Tras el I I-S, el vínculo causal entre pobreza y mala gobernanza con el conflicto fue reinventado para dar explicaciones del terrorismo en los países del $\operatorname{Sur}^{27} y$, en consecuencia, las políticas de desarrollo se redefinieron en clave antiterrorista ${ }^{28}$. La narrativa conservadora impulsada por EEUU trató de subordinar la seguridad humana a las necesidades de pacificación de los países considerados una amenaza ${ }^{29}$, contribuyendo a la debilitación del concepto, a su instrumentalización y, en última instancia, a su progresivo desuso.

Otra de las tendencias que se asentaron con frecuencia tras el II-S fue la explícita alusión al nexo seguridad-desarrollo ${ }^{30}$. Esta idea, más que novedosa, venía a reforzar la tendencia preexistente de vincular la seguridad al desarrollo y viceversa. Bajo este prisma, el Norte Global podía interpretar que en contextos donde ni la seguridad ni el desarrollo fuesen alcanzables el nexo resultaría disfuncional, lo que en última instancia supondría una amenaza para la seguridad nacional de los países desarrollados. Por tanto, la noción del nexo también incidía en la idea del alivio de la pobreza en el Sur Global como medida de contrainsurgencia en el Norte, y el desarrollo y la buena gobernanza, consecuentemente, se convirtieron en instrumentos para contener el "peligro" que representaban las condiciones de los países del Sur ${ }^{3 !}$.

De esta manera, el potencial normativo de la seguridad humana quedó en buena medida reducido. Cierto es que, en general, los Gobiernos estatales y las organizaciones internacionales asumieron visiones más amplias y desarrollistas de la seguridad, pero fue compatible con un refuerzo de la noción de seguridad nacional en los países occidentales ${ }^{32}$. El nexo seguridad-

\footnotetext{
25 SANHAUJA et.al., "El nexo seguridad..." op.cit. p.44.

26 DUFFIELD, M., “Human Security and the Development-Security Nexus. An historical overview”, en Region pratica, 48, junio, 20 I 7, pp.6I-75.

27 BUUR, L. et.al., The Security-Development nexus. Expressions of sovereignty and securitization in Southern Africa. Nordiska Afrikainstitute, HSRC Press , Cape Town, 2007

28 SANHAUJA, J., et.al. "el nexo seguridad-desarrollo..., op.cit. p.60.

29 Tras el II-S el concepto de estados fallidos cobró protagonismo al convertirse en una amenaza para Occidente. Por ejemplo, Afganistán, paradigma de estado fallido, se convirtió el principal receptor de Ayuda Oficial al Desarrollo en la década de los 2000. En CALL, Charles, “The Fallacy of the "Failed State"” en Third World Quarterly,Vol.29, n.8, 2008.

30 Un nexo es una red de conexiones entre ideas, procesos u objetos separados que implica un número indefinido de posibles vínculos y relaciones. En STERN, Maria, OJENDAL, Joakim, "Exploring the security-development nexus", en ARMER, Ramses, OJENDAL, J., SWAIN, Ashok, The Security-development Nexus: Peace, Conflict and Development, Athem Press, Londres, 20I3, p.27.

31 Ibídem. p. 18

32 O, alternativamente, como propone Sanhauja "seguridad internacional”, como suma de la seguridad nacional de determinados países. En SANHAUJA et.al., "El nexo seguridad..." op.cit. p.32.
} 
desarrollo se convirtió en un paradigma utilitario que conllevó, por un lado, la primacía del ámbito de la seguridad, al quedar subordinada la agenda de desarrollo a la política exterior y de defensa de los países occidentales ${ }^{33}$.Y, por otro lado, la elusión del cuestionamiento de las estructuras de poder estatales, pues más allá de aspirar al refuerzo de las instituciones, las políticas impulsadas no implicaban una crítica profunda ni a estas ni al estado ni a las relaciones socioeconómicas imperantes dentro de este ${ }^{34}$. Los Gobiernos eran un interlocutor necesario y se trabajaba a través de instituciones existentes a fin de afrontar las manifestaciones de inseguridad, lo que acabó reforzando las estructuras que contribuían a esta ${ }^{35}$.

Este abandono del ideal de la seguridad humana en su versión amplia prevaleció con la aparición del concepto de resiliencia, que ha adquirido gran popularidad y relevancia, particularmente en el último lustro. Proveniente de otros ámbitos científicos ${ }^{36}$, una de sus características es que resulta muy vago y maleable, lo que lo hace también fácilmente adaptable a contextos muy diversos ${ }^{37}$. Para uno de los autores más prolíficos en la materia, David Chandler, la resiliencia es la capacidad interna de las sociedades para lidiar con las crisis, poniendo el énfasis en el desarrollo de la autoorganización y de las capacidades internas más que en la provisión externa de ayuda, recursos o soluciones políticas ${ }^{38}$.

La emergencia de la resiliencia como paradigma vino a demostrar el fracaso de las estrategias intervencionistas top-down — de arriba hacia abajo- de los noventa y los dos mil, que pretendían mediante la coerción y la condicionalidad instalar un modelo de gobernanza liberal a imagen y semejanza de Occidente ${ }^{39}$. Una de sus novedades es que es un concepto orientado a las sociedades más que a los estados, al seguir una lógica de abajo hacia arriba que, no obstante, es compatible, teóricamente, con el tradicional modelo de arriba hacia abajo. A pesar de que sus partidarios lo encuentran más cercano al concepto de la seguridad humana original ${ }^{40}$, al no poner el foco en la seguridad estatal, sus críticos consideran que esta reorientación ha trasladado la responsabilidad del desarrollo a las comunidades locales y ha servido de justificación para los recortes del presupuesto destinado al desarrollo en tiempos de austeridad ${ }^{41}$. Asimismo, también se reprocha que la atención haya dejado de ponerse en las causas estructurales y para trasladarse a cómo lidiar con las consecuencias, evitando, una vez más, cuestionar el sistema de gobierno neoliberal, y contribuyendo al progresivo abandono de la democratización y el respeto a los derechos humanos como objetivos para alcanzar el desarrollo y la seguridad ${ }^{42}$.

\footnotetext{
33 Ibídem, p.33.

${ }^{34}$ BELLAMY,Alex, MCDONALD, MATT,“The Utility of Human Security”:Which Humans? What Security? A Reply to Thomas \& Tow”, en Security Dialogue, vol.33, 3, 2002, p.373.

${ }_{35}$ PÉREZ DE ARMIÑO, K., ¿Más allá de la seguridad humana?... op.cit.

${ }^{36}$ La psicología fue el ámbito pionero en utilizar la resiliencia, referida a la capacidad de los individuos para vencer las adversidades. También se ha aplicado en ciencias sociales, medioambientales, ingeniería y sistemas socio-ecológicos. BROWN, Katrina, Resilience, development and global change, Nueva York, Routledge, 2016.

${ }^{37}$ JUNCOS, Ana, "Resilience as the new EU foreign policy: a pragmatist turn?", en European Security, 26: I, 20I7, I- I8.

${ }^{38}$ CHANDLER, D., "Rethinking the Conflict-Poverty Nexus: From Securitising Intervention to Resilience”, en Stability: International Journal of Security and Development, 4, pp. I-14, 2015

39 Ibídem.

${ }^{40}$ CHANDLER, D., “Resilience and human security:The post-interventionist paradigm” en Security Dialogue , 43(3), 20I2, 2 I3-229.

4I WAGNER, Wolfgan, ANHOLT, Rosanne, "Resilience as the EU Global Strategy's new leitmotif: pragmatic, problematic or promising?", en Contemporary Security Policy, 37:3, 2016, 414-430 p. 416.

${ }^{42}$ SCHMIDT, Jessica, "Intuitively neoliberal? Towards a critical understanding of resilience governance" en European Journal of International
} 


\section{La evolución del concepto de la seguridad humana en el acervo estratégico de la Unión Europea}

Desde los albores del siglo XXI, la Unión Europea mostró su compromiso por adoptar una visión amplia de la seguridad, ilustrado en la recurrencia al nexo seguridad-desarrollo en sus documentos oficiales. Sin embargo, este hecho no ha implicado la incorporación de la seguridad humana como un pilar básico de su narrativa estratégica, a pesar de que parte de sus principios la subyacen implícitamente.

Los ejemplos de esta indefinición se han sucedido en cada estrategia publicada, lo que ha contribuido a añadir ambigüedad al uso del concepto de la seguridad humana y a coartar desde el inicio su potencial normativo. En 2003, la primera Estrategia Europea de Seguridad (EES) acogía la noción de que "la seguridad es una precondición del desarrollo" y concebía a los estados fallidos como una de las "amenazas clave" para la Unión ${ }^{43}$. Sin embargo, la EES ni siquiera mencionó en ninguna ocasión el concepto de seguridad humana.Además, si bien es cierto que iba en consonancia con determinados preceptos de este, se mostró elusiva respecto a otros de ellos, particularmente los correspondientes a las condiciones democráticas y de derechos humanos. De este modo, la tarea fijada por la UE se limitó simplemente a "promover un anillo de países bien gobernados desde el este hasta el Mediterráneo" ${ }^{44}$, sin precisar en las condiciones que necesitaba un estado para estar "bien gobernado".

En los años posteriores a la Estrategia Europea de Seguridad, dos grupos de estudio externos a la Unión, bajo requerimiento del Alto Representante Javier Solana, presentaron sendos informes sobre las capacidades de la UE en el ámbito de la seguridad. Ambos exhortaron a los decisores europeos a adoptar el concepto de seguridad humana como doctrina estratégica en su política exterior y de seguridad. El primero de ellos, de 2004, el informe de Barcelona $A$ Human Security Doctrine for Europe, esgrimía que la política de seguridad de la Unión debería estar construida sobre la seguridad humana entendida como la libertad de los individuos frente a las inseguridades básicas causadas por las violaciones de derechos humanos ${ }^{45}$. En la misma línea, el segundo de ellos, de 2007, conocido como el informe de Madrid y titulado A European way of security, instaba a definir una manera distintiva de seguridad europea basada en los principios de la seguridad humana ${ }^{46}$.

Es difícil medir cuán exitosos e influyentes fueron estos documentos, pero lo cierto es que tras ellos la Unión comenzó a incorporar, aunque de manera muy tímida y sucinta, el concepto de seguridad humana. Tras el informe de Barcelona, el Consenso Europeo sobre Desarrollo recogía que la Unión apoyará "la seguridad humana de las personas en situación de pobreza" ${ }^{7}$. Sin embargo, esta primera aproximación a la seguridad humana resultaría muy accesoria y limitada, dado que ocupaba una posición marginal en el documento - solo es mencionada dos veces-y

Relations, Vol.2I, Issue 2, 2015.

${ }^{43}$ CONSEJO EUROPEO, A secure Europe in a better World. European Security Strategy. Bruselas, I2 de diciembre de 2003.

44 Ibídem.

45 ALBRECHT, Ulrich, et. al,. A human security doctrine op.cit.

46 ALBRECHT, U., et.al., A European Way of Security. The Madrid Report of the Human Security Study Group comprising a Proposal and Background Report, Human Security Study Group, Madrid, 2007.

47 DIARIO OFICIAL DE LA UNIÓN EUROPEA (DOUE), Declaración conjunta sobre la política de desarrollo de la UE “El Consenso Europeo sobre Desarrollo", 24/02/2006 C 46/0I 
quedaba confinada al ámbito de severa vulnerabilidad. Por el contrario, lo más notorio de este documento es que resulta una contrapartida a la EES. Si esta última manifestaba una concepción de la seguridad vinculada al desarrollo, el Consenso Europeo sobre Desarrollo reforzó este nexo al incorporar cuestiones de seguridad. En concreto, el documento establecía que la Unión redoblaría "sus esfuerzos en materia de prevención de conflictos" y respaldaría "la prevención de la fragilidad del estado mediante reformas en materia de gobernanza, el estado de derecho, medidas de lucha contra la corrupción y la creación de instituciones estatales viables"48. Más adelante, en el documento se aseveraba que "no habrá desarrollo ni erradicación de la pobreza sin paz ni seguridad, y sin desarrollo ni erradicación de la pobreza no habrá paz duradera"49.

Por otro lado, meses después del Informe de Madrid, en 2008, en otro informe elaborado por el Consejo Europeo sobre la implementación de la EES, la UE por primera vez admitía que había trabajado con el propósito de "construir seguridad humana" 50 . En la última parte del informe, además, se instaba a mantener "un enfoque basado en las personas coherente con el concepto de seguridad humana"5I en las actividades de la PESC. No solo era la primera gran evidencia de aceptación e internalización del concepto por parte de la Unión ${ }^{52}$, sino también la primera vez que el Consejo mostraba un compromiso firme con el concepto. No obstante, la seguridad humana quedó lejos de resultar una prioridad estratégica dentro de la PESC según este documento, que solo la menciona de manera escueta en dos ocasiones. Por el contrario, en línea con el Consenso sobre Desarrollo, el informe sí que otorgaba una mayor preponderancia al nexo seguridad-desarrollo, al que mencionaba de manera explícita como una línea estratégica para "construir estabilidad en Europa y más allá", dado que "no puede haber desarrollo sostenible sin paz ni seguridad, y sin desarrollo y erradicación de la pobreza no habrá paz sostenible" 53 .

Sin embargo, la recurrencia del nexo seguridad-desarrollo también decaería paulatinamente en los documentos de la Unión, y la nueva Estrategia Global para la Política Exterior y de Seguridad de la Unión Europea, publicada en 2016, puso en evidencia su relevación por el concepto de resiliencia. Este documento, a diferencia de su predecesora de 2003, sí mencionaría la seguridad humana. De hecho, la UE reconocía tenerla como objetivo complementario del renovado "enfoque integral" a los conflictos: "cuando surgen conflictos violentos [...], la UE se involucrará en una manera práctica de construcción de paz, y fomentará la seguridad humana mediante un enfoque integral" ${ }^{54}$. Más tarde, también reconoce que ante la relación que guardan las amenazas exteriores e interiores, existe "un interés mayor en prevenir conflictos, promover la seguridad humana y abordar las causas radicales de inestabilidad" 55 .

Sin embargo, a pesar del aparente mayor peso otorgado a la seguridad humana en

\footnotetext{
48 Ibídem, p.46/4.

49 lbíd.

${ }^{50}$ CONSEJO EUROPEO, Report on the Implementation of the European Security Strategy - Providing Security in a Changing World -, Bruselas, diciembre de $2008,5407 / 08$, p.2.

51 Ibídem, p. 10.

${ }^{52}$ CHRISTOU, G., “The European Union's human security...” op.cit.

${ }^{53}$ CONSEJO EUROPEO, Report on the... op.cit.p. 8.

${ }^{54}$ SERVICIO EUROPEO DE ACCIÓN EXTERIOR (SEAE), Estrategia Global para la Política Exterior y de Seguridad de la Unión Europea, 2016 , p.9.

55 Ibídem, p. 14.
} 
comparación con los documentos anteriores, el concepto sigue resultando secundario y reducido a la versión restringida, al quedar de nuevo asociado a situaciones de extrema inseguridad. Además, en este caso se ve fagocitado por el de resiliencia, que es mencionado - junto con resiliente - hasta en cuarenta ocasiones, definida como "la capacidad de los estados y las sociedades para reformarse, aguantando así los desastres, y para recuperarse de las crisis internas y externas" 5657 . Si bien la irrupción de este concepto es anterior, la Estrategia Global le otorga una relevancia inusitada. Por un lado, se convirtió en una parte esencial del giro pragmático que la UE pretendía dar a su política exterior"58:"un pragmatismo basado en principios orientará nuestra acción exterior en los próximos años" 59 . Por otro, la introducción de este concepto conllevó la modificación del tradicional nexo seguridad-desarrollo, al invertirse la relación de causalidad entre ambos conceptos ${ }^{60}:$ "los estados son resilientes cuando las sociedades sienten que viven cada vez mejor y tienen esperanza en el futuro". Esta concepción, unido a la novedad de simultanear los enfoques de arriba hacia abajo y de abajo hacia arriba, también se percibe posteriormente en una aseveración que incluye la democracia como virtud de un estado resiliente:

La UE se propone fomentar la resiliencia en las regiones vecinas. Un estado resiliente es un estado seguro, y la seguridad es esencial para la prosperidad y la democracia, pero también es cierto lo contrario. [...] Una sociedad resiliente en la que se den la democracia, la confianza en las instituciones, y el desarrollo sostenible constituye el núcleo de un estado resiliente ${ }^{6 !}$.

\section{La estrategia de la UE hacia el Sahel: la irrelevancia de la seguridad humana ante el nexo seguridad-desarrollo y la resiliencia}

El Sahel se ha convertido en la última década en una de las regiones donde la Unión Europea ha incidido con mayor vigorosidad en el ámbito de la seguridad y el desarrollo. En línea con la concepción de peligrosidad de los estados frágiles para Occidente y a raíz del incremento de conflictividad en la región ${ }^{62}$, en los últimos años de los dos mil la preocupación europea sobre

56 lbíd., p. 23.

57 Ciertamente no era la primera ocasión en la que la Unión Europea introdujo este concepto. En 20I2, la Comisión elaboró el “Enfoque de la Unión Europea para la resiliencia”, una comunicación al Parlamento y el Consejo que fue sucedida por "Las conclusiones del Consejo sobre el enfoque de la UE para la resiliencia". Tras ellos, la Comisión elaboró el documento de trabajo "Plan de Acción para la resiliencia en países propensos a las crisis 2013-2020".

58 JUNCOS, Ana, “Resilience as the new..." op.cit.

59 SEAE, Estrategia Global... op.cit. p. 12.

60 WAGNER,W., ANHOLT, R., “Resilience as the EU...” op.cit. p. 416.

61 SEAE, Estrategia Global... op.cit. p.23.

62 En Mauritania se produjeron dos golpes de estado: en 2005 y, tras una breve experiencia democrática, también en 2008. Su perpetrador Mohamed Uld Abdelaziz, acabó siendo apoyado por la Unión Europea tras su controvertida victoria en las elecciones de 2009 ("EU resumes development cooperation with Mauritania" en Relief Web, 25/0I/20I0, disponible en: https://reliefweb.int/report/mauritania/eu-resumesdevelopment-cooperation-mauritania, última consulta el 21/II/20I9). En Níger, de 2007 a 2009 aconteció la segunda rebelión tuareg protagonizada por el Movimiento de Nigerinos por la Justicia, que entre otras cuestiones reclamaba un reparto más equitativo de los beneficios del uranio, de cuya explotación la empresa francesa Areva ostentaba el monopolio (GRAHAM, Franklin, What the Nigerien coup d'état means to the world Review of African Political Economy Vol. 37, No. I 26, 20 I0, pp. 527-532,y FLYNN, Daniel, DE CLERQ, Geert, Atomic Split. Areva in Niger, Reuters, Special report, 2014). En 2010, se produjo un golpe de estado que depuso al Gobierno e instauró una junta que fue aceptada por la UE y particularmente por Francia, que en el año previo había tenido una serie de desavenencias con el Presidente Tandja a causa del uranio (en BAUDAIS,Virginie and CHAUZAL, Grégory, "Briefing: the 2010 coup d'état in Niger: a praetorian regulation of politics?" en African Affairs Vol. I I 0, No. 439 (abril 20I I), pp. 295-304).Por su parte, Chad estaba inmerso en una guerra civil (2005 - 20I0), así como sus vecinos Sudán y la República Centroafricana. En el caso chadiano, el Presidente Idriss Deby logró mantenerse en el poder con la ayuda del ejército francés (BONO, Giovanna, "The EU's Military Operation in Chad and the Central African Republic:An Operation to Save Lives?" en Journal of Intervention and Statebuilding, 5:I, 23-42, 20I I). Por último, el norte de Malí se había convertido en un lugar propicio para el merodeo de redes terroristas originadas en la guerra civil argelina (I99| - 2002), como el Grupo Salafista para la Predicación y el Combate, que refundado en 2007 como Al Qaeda en el Magreb Islámico (AQMI) secuestró en la región hasta a 25 ciudadanos europeos entre 2008 y 20 I I (En DÍEZ, Jesús, “Desafíos de seguridad en el Sahel: conflictos armados y terrorismo yihadista” en Cuaderno de Estrategia I76 del IEEE, 
esta se acrecentó ${ }^{63}$. En $201 \mathrm{I}$, el recién inaugurado Servicio Europeo de Acción Exterior lanzó su primera estrategia para la seguridad y el desarrollo de una región específica: la Estrategia para la Seguridad y el Desarrollo en el Sahel. Esta vendría a inaugurar unos años de sucesivas iniciativas intervencionistas por parte de Bruselas, lo que ha convertido a la región africana en un ejemplo paradigmático de la aplicación de las diferentes doctrinas estratégicas adoptadas por la Unión en su acervo estratégico. Por tanto, la evolución de las políticas europeas hacia el Sahel sirve para ilustrar la inicial preponderancia del ambiguo nexo seguridad-desarrollo; la escasa relevancia estratégica del concepto de la seguridad humana; $y$, posteriormente, la irrupción de la resiliencia como paradigma alternativo.

En primer lugar, en el primer párrafo de la Estrategia para la Seguridad y el Desarrollo de 201 I ya se recoge que "la seguridad y el desarrollo en el Sahel no pueden ser separados" y que “la UE tiene un gran papel que desempeñar para conseguir un entorno más seguro en la región, donde el desarrollo pueda tener lugar y donde los intereses de los ciudadanos de la Unión estén también protegidos" ${ }^{4}$. Esta afirmación inicial denota, por un lado, la justificación de la idoneidad de la intervención europea en el Sahel; $y$, por otro lado, la subordinación de las iniciativas en la región a las prioridades de la propia seguridad de la $U E^{65}$. Otras partes del texto invitan a reforzar esta idea, pues el objetivo común de la coordinación europea debía ser "reforzar la seguridad y el desarrollo — del Sahel- para así fortalecer la seguridad de la propia Unión"66. Además de ello, la Estrategia destaca por el escaso empleo tanto del concepto de la seguridad humana, solo mencionado en una ocasión, como el de la resiliencia, también nombrado una vez. En cuanto a la seguridad humana, aparece en referencia al desafío que representaban la falta de seguridad y de estado de derecho: "estos Estados — sahelianos - tienen insuficientes capacidades [...] para asegurar la seguridad humana" ${ }^{67}$. En este caso, vuelve a observarse la asociación del concepto a situaciones de vulnerabilidad extrema, $y$, además, queda subordinado a la capacidad de provisión de los estados. Por su parte, el concepto de resiliencia es utilizado como parte de la estrategia de lucha contra el extremismo violento y la radicalización, dado que la UE aspiraba a "reforzar la resiliencia de las sociedades para contrarrestar el terrorismo" 68 .

Sin embargo, poco después de la publicación de la Estrategia, el panorama regional cambió drásticamente. En el contexto de las revueltas árabes de 201 I, Libia se sumió en una guerra civil en la que más de una decena de países de la UE participaron en el marco de una intervención militar coordinada por la OTAN y avalada por Naciones Unidas ${ }^{69}$. Esta intervención, si bien contribuyó al derrocamiento de Muamar el Gadafi, no propició la pacificación del país. Al contrario, el Estado

\footnotetext{
2015, Ministerio de Defensa, Madrid; y ECHEVERRÍA, Carlos, AQMI y otros grupos de su entorno: una compleja red de carácter violento, Documento de investigación 02/20I3 IEEE, 20I3, Madrid).

${ }^{63}$ PARLAMENTO EUROPEO, A Coherent EU Strategy ... op.cit.

${ }^{64}$ SEAE, Strategy for security and development in the Sahel, Bruselas, $201 \mathrm{I}$.

${ }^{65}$ MARÍN, Ainhoa, "Políticas de la Unión Europea en el Sahel: una mirada económica para un enfoque más allá de la seguridad" en PUIG, Oriol y ROCA,Albert (eds.), El Sahel de las gentes. Más allá del síndrome de la seguridad, Monografías CIDOB, Barcelona, 2019.

${ }^{66}$ Ibídem, p.2.

67 Ibíd., p.3.

${ }^{68}$ Ibíd., p.7.

${ }^{69}$ Avalada por la resolución 1973 del Consejo de Seguridad de Naciones Unidas. Liderada por Reino Unido, Francia y EEUU, en la intervención también participaron, con menor grado de implicación, estados miembro como Dinamarca, Italia, Bélgica, Países Bajos, Polonia, España, Suecia, Grecia, Rumanía y Bulgaria. LINDSTRÖM, Madeleine, ZETTERLUND, Kristina, Setting the Stage for the Military Intervention in Libya, FOI, R3498-SE, Suecia, 2012.
} 
libio se desmoronó, agravando la inestabilidad de la región del Sahel, y el conflicto se reavivó a partir de 2014. Tras la derrota de Gadafi, rebeldes tuaregs del norte de Malí se beneficiaron tanto del retorno de combatientes como del saqueo del arsenal del Ejército libio $y$, unidos en el Movimiento de Liberación Nacional de Azawad, se levantaron en armas para proclamar la independencia de la región septentrional del país en abril de $2012^{70}$. Un mes antes, en la capital, Bamako, se produjo un golpe de estado militar que derrocó al Gobierno democrático vigente en marzo ${ }^{71}$. Posteriormente, en junio, los yihadistas de Ansar Dine cooptaron la rebelión del MLNA, proclamaron la sharia en Azawad, y, en enero de 20I3, lanzaron una ofensiva contra Bamako, a la que Francia respondió militarmente con la Operación Serval ${ }^{72}$.

Esta sucesión de acontecimientos hizo reaccionar a la UE. En agosto de 2012 Bruselas lanzó la misión civil para la construcción de capacidades EUCAP Sahel Níger ${ }^{73}$, y en diciembre, el Consejo Europeo aprobó la misión militar para contribuir a la formación de las Fuerzas Armadas de Malí, EUTM Mali ${ }^{74}$. Además, ese mismo mes se lanzó la Alianza Global para las Iniciativas de Resiliencia en el Sahel y África occidental (AGIR), que reunía a Gobiernos, organizaciones regionales, donantes y ONG "para el refuerzo de la nutrición, de la agricultura, la productividad y la gobernanza alimentaria"75. Esta iniciativa supuso la puesta en marcha del todavía incipiente concepto de resiliencia ${ }^{76}$, que en este caso quedaba condicionado por el grave contexto de crisis de nutrición y alimentos presente en la zona ${ }^{77}$.

En la práctica, estos cambios en la estrategia y el discurso de la Unión propiciaron que los asuntos de seguridad se inmiscuyeran en los de desarrollo, siendo un claro ejemplo de ello las modificaciones realizadas en los respectivos Planes Indicativos Nacionales (PIN) elaborados por Bruselas para cada país. Con la renovación del presupuesto europeo en 2014 y, consecuentemente, la renovación del propio Fondo Europeo de Desarrollo - encargado de financiar los PIN-, tanto el desembolso como el destino de las partidas asignadas a los países sahelianos variaron sustancialmente. Quedó confirmado que determinadas partidas tradicionalmente relacionadas con el ámbito de la seguridad habían quedado enmarcadas en los dominios del FED. En primer lugar, las partidas correspondientes a la gobernanza, presentes en el PIN 2009-20I3 de cada país, quedarían sustituidas y ampliadas bajo la nomenclatura de "refuerzo del estado de derecho" en cuatro de los cinco países - la excepción fue Burkina Faso-. Esta sección pasó a ser la principal beneficiaria del $1 I^{\circ}$ FED en los casos de Níger —en el que aparece desglosada en tres

70 DÍEZ, J., “Malí, obstáculos y respuestas para enfrentar una amenaza compleja”, en Panorama geopolítico de los conflictos, IEEE, Madrid, 20 I8.

71 lbídem.

72 Ibídem.

${ }^{73}$ Lanzada en agosto de 2012 tras la Decisión 2012/392/PESC del Consejo, se convirtió en la segunda misión EUCAP de la historia, tras la de Somalia, establecida apenas días antes.

${ }^{74}$ CONSEJO DE LA UE, Main results of the 3209th Council meeting (en línea), Bruselas, I0 de diciembre, p.16. Disponible en: https://goo.gl/ T4YLWD. Última consulta: I8/09/2019.

75 COMISIÓN EUROPEA, AGIR- Building resilience in the Sahel and West Africa. ECHO Factsheet. (En línea). Disponible en: https://goo.gl/kY2JDC. Última consulta: $18 / 09 / 2019$

76 Definido como "la capacidad de hogares, familias y sistemas vulnerables de hacer frente a la incertidumbre y al riesgo de shocks, de resistir y responder efectivamente a los shocks, así como de recuperarse y adaptarse de una manera sostenible”. Ibídem.

77 Según la UE, las crisis de nutrición y alimentos se han sucedido en los años 2005, 2008, 2010,2012, 20I7, 2018 y 20I9. En 20I2 se estimaba que I8,7 millones de personas estaban en situación de inseguridad alimentaria. En COMISIÓN EUROPEA, 2012 Sahel Food \& Nutrition Crisis: ECHO's response at a glance. Disponible en: https://ec.europa.eu/echo/files/aid/countries/ECHO_20I2_Response_Sahel_Crisis_en.pdf . Última consulta: $21 / 1 \mathrm{l} / 2019$. 
subpartidas $^{78}$ - y Malí y la segunda partida en importancia en el caso de Mauritania.

El sector concerniente al "refuerzo del estado de derecho", según los PIN, quedó ampliado con objetivos que se expresaban como prioritarios en los tres países objeto de la Estrategia: el control efectivo del territorio y la lucha contra el crimen organizado —en el caso mauritano_- ${ }^{79}$; la seguridad, la consolidación de la paz y el apoyo a las regiones afectadas por conflictos —según el PIN nigerino — ${ }^{80}$; y la restauración de la seguridad, el refuerzo de las capacidades de las fuerzas y de los sistemas de seguridad —en el caso de Malí—81. Junto a ello, el PIN de Chad también contiene como un objetivo prioritario "contribuir a la consolidación de la paz" y el "refuerzo de la seguridad ante una situación de fragilidad" 82 , mientras que el de Burkina Faso, único país que conservó la sección de "gobernanza" en el PIN de 2014, incluyó como novedad el objetivo, dentro de esta, del refuerzo del estado de derecho y los mecanismos de seguridad ${ }^{83}$. Estas transformaciones no solo fueron nominales, sino cuantitativas. La cuantía destinada al refuerzo del estado de derecho superó con creces aquella destinada a la gobernanza en todos los países menos Chad ${ }^{84}$.

Esta preeminencia de la seguridad se afianzaría con la publicación del Plan de Acción Regional para el Sahel (20I5-2020) (PAR), en el que tres de las cuatro áreas específicas de las que consta están claramente relacionadas con esta ${ }^{85}$. No obstante, el PAR, que afirmaba que "la seguridad y el desarrollo en la región del Sahel son cruciales para la Unión Europea"86, también supuso la irrupción del concepto de resiliencia en la estrategia europea para con la región saheliana. Si bien no alcanza el rango de objetivo prioritario, el concepto de resiliencia aparece en el documento hasta en veinticinco ocasiones, lo que contrasta, en primer lugar, con la única mención recibida en la predecesora Estrategia para el Sahel de 20I I; y, en segundo lugar, con las escuetas dos veces en las que la seguridad humana es mencionada en el propio PAR. El propio documento admite que "el objetivo estratégico original de la Estrategia para el Sahel de 20I I, enfatizando el nexo seguridaddesarrollo y sus pilares para su implementación, siguen siendo plenamente pertinentes" ${ }^{\text {" } 7 \text {. Es }}$ por ello que esta estrategia puede considerarse una transición entre este paradigma y el de la resiliencia, que ocupa un lugar muy relevante en las estrategias planeadas para reforzar las cuatro líneas de acción existentes.

Además del PAR, en 2015 también verían la luz la segunda misión civil de la UE en la región, la EUCAP Sahel Malí,y el nuevo Fondo Fiduciario de Emergencia para África (EUTF, por sus siglas en inglés), con la misión de "dotar de una respuesta integrada y coordinada a las diferentes causas

\footnotetext{
${ }^{78}$ Las tres partidas que aparecen en lugar de "refuerzo al estado de derecho" en este país son: apoyo a las regiones afectadas por el conflicto y la inseguridad; seguridad, gobernanza y consolidación de la paz; y el refuerzo de las capacidades del Estado para la provisión de políticas sociales.

79 COMISIÓN EUROPEA, Mauritania. Programme Indicatif National 20I4-2020, 2014

80 COMISIÓN EUROPEA, Niger. Programme Indicatif National 2014-2020, 2014.

81 COMISIÓN EUROPEA, Mali. Programme Indicatif National 2014-2020, 2014.

${ }^{82}$ COMISIÓN EUROPEA, Tchad. Programme Indicatif National 2014-2020, 2014

${ }^{83}$ COMISIÓN EUROPEA, Burkina Faso. Programme Indicatif National 2014-2020, 2014.

${ }^{84}$ Datos extraídos de los PIN de los cinco países sahelianos citados con anterioridad.

${ }^{85}$ Las áreas son: prevención y la lucha contra la radicalización; creación de condiciones apropiadas para la juventud; migraciones, la movilidad y la gestión fronteriza; y la lucha contra el tráfico ilícito y el crimen organizado transnacional. CONSEJO DE LA UE, EU Sahel strategy. op.cit. p.3.

${ }^{86}$ CONSEJO DE LA UE, EU Sahel strategy. op.cit. p.4.

${ }^{87}$ Ibídem, p.3.
} 
de la inestabilidad, la migración irregular y los desplazados forzados"88. El Sahel, junto con el lago Chad, es una de sus tres áreas de acción, además del norte de África y el Cuerno de África. Hasta la elaboración de este artículo, cuatro mil millones y medio de euros habían sido asignados por el EUTF, de los cuales un $88 \%$ provenían del FED y de otros instrumentos financieros como el Instrumento de Cooperación al Desarrollo (ICD), el Instrumento Europeo de Vecindad (IEV) o la Dirección General de Ayuda Humanitaria y Protección Civil de la Comisión Europea $(\mathrm{ECHO})^{89}$. El resto es financiado por los países participantes. Los fondos asignados van destinados a cuatro sectores diferentes: el refuerzo de la resiliencia; la gobernanza y la prevención de conflictos; la gestión migratoria; y el desarrollo económico. En los casos de los países del Sahel, gobernanza y prevención de conflictos es el sector que más recursos recibe - $41 \%$-, seguido del refuerzo de la resiliencia $-27 \%{ }^{90}$. Al provenir la financiación de estos proyectos, en su gran mayoría, del FED, encontramos en este fondo fiduciario un nuevo caso de recursos asignados al desarrollo que se destinan, en mayor medida, a asuntos de seguridad. Por tanto, la lógica del nexo seguridaddesarrollo establecida en los documentos oficiales sigue vigente en la práctica, con la novedad de la inclusión de la resiliencia como uno de los principales acaparadores de las estrategias de desarrollo.

Fruto de ello, AGIR, aún vigente, se vio reforzada por una iniciativa más, la Alianza Sahel, creada por la UE, Alemania y Francia en $2017^{91}$ y a la que se sumaron posteriormente el Banco Mundial, el Banco Africano de Desarrollo, España, Reino Unido, Luxemburgo, Italia, Dinamarca y Países Bajos. Paradójicamente, el concepto de resiliencia no ocupó explícitamente un papel central en la fundación de esta alianza ni tampoco en sus objetivos, aunque está orientada a la creación de capacidades en los entornos locales. Dos de sus proyectos tienen como objetivo el refuerzo de la seguridad humana: el concerniente al "Refuerzo de la seguridad humana y la resiliencia comunitaria en el Sahel" y el de la "Gestión fronteriza para la estabilidad y la seguridad humana"92. En el primero de ellos, la seguridad humana y la resiliencia aparecen combinados con el objetivo de prevenir los conflictos locales y mejorar la gestión fronteriza, mientras que en el segundo proyecto, la seguridad humana está encaminada a la "mejora de la seguridad comunitaria y las tensiones locales"93. Como novedad, en estos últimos años - como en los mencionados casos de la Alianza Sahel o el EUTF- se observa la vinculación explícita tanto de la seguridad humana como de la resiliencia al control fronterizo, lo cual no es baladí. Refleja, por un lado, que las dinámicas de peligrosidad asociadas a la pobreza parecen reforzarse con los nuevos conceptos que irrumpen en la agenda europea; $y$, otro lado, que la contención de la inmigración ha pasado a ser una de las prioridades europeas en materia de seguridad.

${ }^{88}$ COMISIÓN EUROPEA, EU Emergency Trust Fund, Comisión Factsheets (En línea). Disponible en: https://goo.gl/QLwrLj. Última consulta el 15/09/2019.

${ }^{89}$ COMISIÓN EUROPEA, EU Emergency Trust Fund for Africa. Financial Resources, (en línea). Disponible en: https://ec.europa.eu/trustfundforafrica/ content/trust-fund-financials_en, última consulta el II/09/20I9.

90 Datos extraídos de la página web oficial del Fondo Fiduciario de Emergencia para África.Disponibles en:https://ec.europa.eu/trustfundforafrica/ region_en. Última consulta el II/09/2019.

91 Aunque su puesta en marcha efectiva no se produjo hasta enero de 2019 , cuando la Alianza anunció la implementación de 730 proyectos hasta 2022, con un presupuesto de II mil millones de euros. Información disponible en su página web https://www.alliance-sahel.org/en/ sahel-alliance/. Última consulta: I7/09/20I9.

92 Información disponible en https://www.alliance-sahel.org/en/the-projects/. Última consulta: I7/09/2019.

93 Página web de la Alianza Sahel. Información sobre el proyecto: https://www.alliance-sahel.org/en/projects/border-management-for-stabilityand-human-security/. Última consulta: I7/09/2019. 
De este modo, es especialmente significativo que la proliferación del concepto de la resiliencia en los últimos años se haya producido en paralelo al progresivo deterioro de la seguridad en el Sahel. Tras las sucesivas intervenciones e iniciativas europeas, en Malí y Burkina Faso las milicias armadas han proliferado94; mientras que el conflicto en Libia conllevó el desgobierno y la falta de control fronterizo, aumentándose, inicialmente, los flujos migratorios hacia Europa desde este país ${ }^{95}$, provenientes en su gran mayoría desde Níger ${ }^{96}$. Este país se ha convertido en un ejemplo paradigmático de la anteposición, por parte de la UE, del control migratorio, hasta el punto de condicionar la ayuda al desarrollo al buen desempeño de este país a la hora de contener los flujos de migración ${ }^{97}$.

Ante este contexto, la adopción del concepto de resiliencia cobra mayor utilidad para la UE, pues el hecho de poner el foco en cómo los estados y las sociedades lidian con los desastres y las crisis internas y externas permite desviar la atención de su parte de responsabilidad en los acontecimientos sucedidos en la región del Sahel en los últimos años.Además, del mismo modo, le posibilita eludir el cuestionamiento tanto de la coherencia como de la efectividad de sus estrategias. A pesar de la pretendida multidimensionalidad, en su empeño por reducir la inseguridad, contener los flujos de migración y contribuir al desarrollo en el Sahel, Bruselas ha privilegiado unos ámbitos por encima de otros. Ello ha propiciado unos efectos secundarios que evidencian la importancia de cubrir integralmente las dimensiones originales de la seguridad humana: económica, alimentaria, sanitaria, ambiental, personal, comunal y política.

En primer lugar, en Malí, Níger o Burkina Faso, a pesar del esfuerzo europeo, no solo se han producido más ataques contra civiles con el paso de los años ${ }^{98}$, sino que ha crecido la violencia intercomunitaria e interétnica ${ }^{99}$. En estos conflictos, a menudo entre agricultores y ganaderos ${ }^{100}$, también influye el deterioro de las condiciones medioambientales en una región muy afectada por el cambio climático ${ }^{101}$ y las restricciones de la movilidad en la región ${ }^{102}$. En Níger, precisamente la interrupción de rutas migratorias ancestrales hacia Libia u otros países ha propiciado una mayor peligrosidad y coste para los migrantes, así como la vulneración de sus derechos humanos, en una zona tradicionalmente de tránsito ${ }^{103}$. Además, los movimientos de personas han sido un sustento económico para gran parte de la población de la zona norte nigerina, por lo que existen multitud de ciudadanos agraviados a los que no les resulta fácil encontrar una alternativa plausible para

\footnotetext{
${ }^{94}$ LEBOVICH, Andrew, Mapping Armed Groups in Mali and the Sahel, ECFR. Disponible en: https://www.ecfr.eu/mena/sahel_mapping. Última consulta $01 / 12 / 2019$.

95 Datos disponibles en la web de la UNHCR: https://data2.unhcr.org/en/situations/mediterranean. Última consulta 29/I I/2019.

${ }^{96}$ Cabe precisar que la gran mayoría de estos migrantes no son originarios de Níger; este país ejerce principalmente de tránsito. En PUIG, Oriol, PÉREZ, Marta, Níger gendarme de Europa, Informe de Oxfam Intermón n 52, julio 2019

${ }^{77}$ Ibídem. También en PRESTIANNI, Sara, Security and migration. Economic interest and violation of basic rights. The cases of Libya, Niger and Egypt, ARCl, 2019. Disponible en: https:/www.arci.it/app/uploads/2019/05/report-2019-inglese-normal.pdf, última consulta el 02//2/2019.

${ }^{98}$ Según los datos de ACLED data, disponibles en acleddata.com. También en ACLED: Political violence skyrockets in the Sahel. Disponible en https://www.acleddata.com/2019/03/28/press-release-political-violence-skyrockets-in-the-sahel-according-to-latest-acled-data/. Ú1tima consulta: $23 / 1 \mathrm{I} / 2019$.

99 EIZENGA, Daniel, “Long Term Trends across Security and Development in the Sahel”, en West African Papers. OECD, 25V, 2019.

${ }^{100}$ Ibídem.

${ }^{101}$ lbíd.

${ }^{102}$ Ibíd.

${ }^{103}$ PUIG, O., PÉREZ, M., Niger gendarme...op.cit
} 
subsistir ${ }^{104}$. Todo lo anterior está ligado a uno de los grandes desafíos, la seguridad alimentaria ${ }^{105}$ y a una mejorable gobernanza. A pesar de la insistencia en el refuerzo de esta por parte de la UE, poco se ha avanzado en la democratización de la región, y en países como Níger o Chad hay evidencias del aumento del autoritarismo ${ }^{106}$. No solo se mantienen las mismas estructuras de poder que existían antes de la primera estrategia europea en la zona, a excepción de Malí, sino que estas han quedado legitimadas por Bruselas, que se sirve de ellas en sus intentos por promover el desarrollo y la seguridad. Por tanto, la seguridad humana es un instrumento teórico que, si bien ha perdido vigencia y relevancia, no ha perdido su idoneidad: en el complejo escenario del Sahel sus preceptos siguen antojándose apropiados, mientras que los posteriores conceptos, al distanciarse de los mismos, se están revelando infructuosos.

\section{Conclusiones}

A pesar de la evolución de la narrativa estratégica de la Unión Europea, existe una continuidad entre los sucesivos paradigmas empleados, más que una disrupción. Si bien los enfoques o ámbitos de incidencia sugeridos por los conceptos han diferido, el punto en común que subyace a todos ellos es su adecuación a los intereses de seguridad de la propia Unión Europea. Esta ha moldeado a su antojo la interpretación teórica de los conceptos y su aplicación en función de sus cambiantes prioridades en el ámbito de la seguridad a lo largo de los años.

Un buen ejemplo de ello lo encontramos en el Sahel. En un primer momento, las necesidades más urgentes por parte de Bruselas en esta región pasaban por la dotación de capacidades a los ejércitos nacionales en aras de combatir las amenazas que le afectaban -como el secuestro de cooperantes europeos o la caída del norte de Malí en manos de grupos armados-, para lo que resultó más propicio recurrir al nexo seguridad-desarrollo e implementar estrategias de intervención de arriba hacia abajo. Posteriormente, estas necesidades se han combinado con otras más recientes como la prevención de la radicalización o la contención de los flujos migratorios que se originan y/o transitan por esta región con Europa como destino. Por ello, para Bruselas ha resultado más apropiada una mayor incidencia en la resiliencia, un enfoque más centrado en las comunidades locales que implícitamente pone en entredicho el éxito de las estrategias anteriores y elude la asunción de responsabilidades en el deterioro de la seguridad en la región.

Por tanto, ante el panorama cambiante de desafíos, cada estrategia europea ha buscado ser más efectiva y pragmática para sus intereses, teniendo como punto de partida la preponderancia de la seguridad sobre el desarrollo, lo que ha resultado en la vinculación de las cuestiones de seguridad a la agenda de los fondos de desarrollo. Sin embargo, entre las herramientas teóricas confiadas para ello, la seguridad humana ha desempeñado un rol muy marginal, como indica su escasa importancia y sus escuetas menciones tanto en los documentos estratégicos más generales de la Unión como en aquellos más concretos, como los Planes Indicativos Nacionales. Además de ello, en las veces en las que se ha aludido a ella, la seguridad humana aparece desvirtuada. En primer lugar, por la ausencia de una definición práctica; en segundo lugar, por quedar reducida a una versión minúscula asociada a vulnerabilidades físicas extremas; $y$ en tercer lugar, porque su

\footnotetext{
${ }^{104}$ HOFFMAN, Anette, et al, Migration and Markets in Agadez. Economic alternatives to the migration industry. CRU Report, 2017.

105 Según la FAO, que posee una concepción más amplia del Sahel, 29 millones de sahelianos sufren inseguridad alimentaria. Disponible en: http:// www.fao.org/emergencies/crisis/sahel/intro/es/. Última consulta el 24/I I/20I9.

${ }^{106}$ EIZENGA, D. “Long term...” op. cit.
} 
provisión ha quedado confiada a Estados que, en ocasiones, contribuyen a la inseguridad —entre ellos, los propios europeos-.

Más que ser suplantada por el nexo seguridad-desarrollo y la resiliencia, la seguridad humana ha seguido conviviendo con estos, aunque de una forma accesoria y poco operativa. Es difícil medir cuán inspiradora ha sido para los paradigmas ulteriores, pero a tenor de lo manifestado por Bruselas se le puede reconocer una cierta influencia en ellos. El nexo seguridad-desarrollo heredó una noción más amplia de la seguridad al incorporar cuestiones de desarrollo, aunque la primacía de esta seguía siendo clara.Asimismo, se centraba eminentemente en la libertad frente al temor mediante un enfoque de arriba hacia abajo que se alejaba del ideal original de la seguridad humana. Por su parte, la resiliencia parece querer recuperar una perspectiva más multifacética y adaptada a las necesidades de los individuos, pero se muestra poco ambiciosa a la hora de procurar transformaciones estructurales.

Veinticinco años después, la seguridad humana apenas representa un vestigio utópico para la Unión Europea, cuyos intentos por acercarlo a la realidad se han demostrado parciales e infructuosos. Los conceptos posteriores han sido consecuencia de esta falta de conciliación entre lo ideal y lo terrenal; $y$ han servido para tratar de paliar la discordancia entre las prioridades de desarrollo de países del Sur y los intereses de seguridad de la Unión Europea, favoreciendo en definitiva a estos últimos.

\section{Bibliografía}

ALBRECHT, U., CHINKIN Cristine, COLLANTES CELADOR, Gemma, FLECHTNER Stefanie, GLASIUS, Marlies, KALDOR, Mary, KILJUNEN, Kimmo, KLABBERS, Jan, KUPER, Jenny, LICHT, Sonia, LOTTI, Flavio, REINHARDT,Klaus, SCHMEDER, Genevieve, SEIFTER, Pavel, SERRA Narcis, WEISSKIRCHEN, Gert, A European Way of Security. The Madrid Report of the Human Security Study Group comprising a Proposal and Background Report, Human Security Study Group, Madrid, 2007.

ALBRECHT, Ulrich, CHINKIN, Christine, DERVIS, Kemal, DWAN, Renata, GIDDENS, Anthony, GNESOTTO, Nicole, KALDOR, Mary, LICHT, Sonia, PRONK, Jan, REINHARDT, Klaus, SCHMEDER, Genevieve, SEIFTER, Pavel and Serra, NARCIS, A human security doctrine for Europe: the Barcelona Report of the Study Group on Europe's Security Capabilities, Study Group on Europe's Security Capabilities, Barcelona, 2004.

ANDERSON, Ruben, et.al., From Hybrid Peace to Human Security: Rethinking EU Strategy towards Conflict, The Berlin Report of the Human Security Study Group, 2016.

BAUDAIS, Virginie and CHAUZAL, Grégory, "Briefing: the 2010 coup d'état in Niger: a praetorian regulation of politics?" en African Affairs Vol. I I 0, No. 439 (abril 20I I), pp. 295-304).

BELLAMY, Alex, MCDONALD, MATT, “The Utility of Human Security”: Which Humans? What Security? A Reply to Thomas \& Tow”, en Security Dialogue, vol.33, 3, 2002, p. 373.

BONO, Giovanna, "The EU's Military Operation in Chad and the Central African Republic: An Operation to Save Lives?" en Journal of Intervention and Statebuilding, 5: I, 23-42, 20I I).

BROWN, Katrina, Resilience, development and global change, Nueva York, Routledge, 2016.

BUUR, Lars, JENSEN, Steffen, STEPPUTAT, Finn, The Security-Development nexus. Expressions of sovereignty and securitization in Southern Africa. Nordiska Afrikainstitute, HSRC Press , Cape Town, 2007.

CALL, Charles, “The Fallacy of the 'Failed State”" en Third World Quarterly,Vol. 29, No. 8, 2008.

CHANDLER, D., "Resilience and human security: The post-interventionist paradigm" en Security Dialogue 43(3) 213 229,2012

CHANDLER, D., "Rethinking the Conflict-Poverty Nexus: From Securitising Intervention to Resilience”, en Stability: International Journal of Security and Development, 4(I), pp. I- I4, 20 I5.

CHRISTIE, Ryerson., “Critical Voices and Human Security: to Endure, to Engage or to Critique?”, en Security Dialogue, n. ${ }^{\circ} 41,2010$.

CHRISTOU, George, “The European Union's human security discourse: where are we now?" en European Security, vol. 23, 20I4-3 PP 364-38I.

COMISIÓN EUROPEA, AGIR-Building resilience in the Sahel andWest Africa. ECHO Factsheet. (En línea). Disponible en: 
https://goo.gl/kY2JDC. Última consulta: I8/09/2019.

COMISIÓN EUROPEA, Burkina Faso. Programme Indicatif National 20/4 - 202020 I 4.

COMISIÓN EUROPEA, Eu Emergency Trust Fund for Africa. Financial Resources, (en línea). Disponible en: https:// ec.europa.eu/trustfundforafrica/content/trust-fund-financials_en, última consulta el II/09/2019.

COMISION EUROPEA, EU Emergency Trust Fund, Comisión Factsheets (En línea). Disponible en: https://goo.gl/QLwrLj. Última consulta el I5/09/2019.

COMISIÓN EUROPEA, Global Alliance for Resilience (AGIR) - Sahel andWest Africa (en línea). Disponible en: https:// ec.europa.eu/knowledge4policy/online-resource/global-alliance-resilience-agir-sahel-west-africa en , última consulta el II/09/2019.

COMISIÓN EUROPEA, Mali. Programme Indicatif National 20I 4 - 2020, 2014.

COMISIÓN EUROPEA, Mauritania. Programme Indicatif National 2014 - 2020, 2014.

COMISIÓN EUROPEA, Niger. Programme Indicatif National 2014 - 2020, 2014.

COMISIÓN EUROPEA, Tchad. Programme Indicatif National 20I 4 - 2020, 2014.

CONSEJO DE EUROPA, Declaration of the Council of Europe's First Summit, Viena, octubre de 1993. Disponible en:https:// www.cvce.eu/en/obj/declaration_of_the_council_of_europe_s_first_summit_vienna_9_october_1993-end7c530b5-a7c9-43f9-95af-c28b3c8b50d3.html. Última consulta el 20/I I/2019.

CONSEJO DE LA UE, Main results of the 3209th Council meeting (en línea), Bruselas, 10 de diciembre, p.I6. Disponible en: https://goo.gl/T4YLWD. Última consulta: I8/09/2019.

CONSEJO DE LA UE, Council conclusions on implementation of the EU Strategy for Security and Development in the Sahel. FA Council Meeting Bruselas, I7/03/2014.

CONSEJO DE LA UE, EU Sahel strategy. Regional Action Plan 20 I5-2020. Bruselas.

CONSEJO EUROPEO, A secure Europe in a better World. European Security Strategy. Bruselas, I 2 de diciembre de 2003.

CONSEJO EUROPEO, Report on the Implementation of the European Security Strategy - Providing Security in a Changing World -, Bruselas, diciembre de 2008, S407/08.

DIARIO OFICIAL DE LA UNIÓN EUROPEA (DOUE), Declaración conjunta sobre la política de desarrollo de la UE "EI Consenso Europeo sobre Desarrollo", 24/02/2006 C 46/0 I.

DÍEZ, Jesús, “Desafíos de seguridad en el Sahel: conflictos armados y terrorismo yihadista” en Cuaderno de Estrategia 176 del IEEE, 20I5, Ministerio de Defensa, Madrid.

DÍEZ, Jesús, “Malí, obstáculos y respuestas para enfrentar una amenaza compleja”, en Panorama geopolítico de los conflictos, IEEE, Madrid, 2018.

DUFFIELD, Mark, “Human Security and the Development-Security Nexus. An historical overview”, en Region pratica, 48, junio, 20I7, pp. 6I-75.

DUFFIELD, Mark, Las nuevas guerras en el mundo global. La convergencia entre desarrollo y seguridad, Catarata, Madrid, 2001

ECHEVERRÍA, Carlos, AQMI y otros grupos de su entorno: una compleja red de carácter violento, Documento de investigación 02/20I3 IEEE, 20I3, Madrid.

EIZENGA, Daniel, “Long Term Trends across Security and Development in the Sahel”, en West African Papers. OECD, $25 \mathrm{~V}, 2019$

En KALDOR, Mary, y RANGELOR, lavor, The Handbook of Global Security Policy, John Wiley \& Sons, 2014

FLYNN, Daniel, DE CLERQ, Geert, Atomic Split. Areva in Niger, Reuters, Special report, 2014

GRAHAM, Franklin, What the Nigerien coup d'état means to the world Review of African Political Economy Vol. 37, No. 126, 2010, pp. 527-532

HELMAN, Gerald y RATNER, Steven, en "Saving Failed States” en Foreign Policy No. 89, pp. 3-20, 1992.

HOFFMAN, Anette, MEESTER, Jos, NABARA, Hamidou, Migration and Markets in Agadez. Economic alternatives to the migration industry. CRU Report, 2017.

JUNCOS,Ana, “Resilience as the new EU foreign policy paradigm: a pragmatist turn?”, en European Security, 26: I, 20 I 7, I- 18.

LEBOVICH, Andrew, Mapping Armed Groups in Mali and the Sahel, ECFR. Disponible en: https://www.ecfr.eu/mena/ sahel_mapping. Última consulta 0I/I2/2019.

LINDSTRÖM, Madeleine, ZETTERLUND, Kristina, Setting the Stage for the Military Intervention in Libya, FOI, R3498SE, Suecia, 2012

MARÍN, Ainhoa, "Políticas de la Unión Europea en el Sahel: una mirada económica para un enfoque más allá de la seguridad" en PUIG, Oriol y ROCA, Albert (eds.), El Sahel de las gentes. Más allá del síndrome de la seguridad, Monografías CIDOB, Barcelona, 2019.

MIHALKA, Michael, “Cooperative Security in the 2 I stCentury”, en Connections Vol. 4, No. 4 , 20I 5, pp. I I 3 - I 22.

NEWMAN, Edward, “Critical Human Security Studies”, en Review of International Studies, n.36, 2010

OECD, Geographical Distribution of Financial Flows to Developing Countries 20I I, OECD publishing, 20I 2.

PARLAMENTO EUROPEO, A Coherent EU Strategy for the Sahel, DG External Policies, Bruselas, 20I 2.

PÉREZ DE ARMIÑO, K., ¿Más allá de la seguridad humana? desafios y aportes de los estudios críticos de seguridad en Cursos de derecho internacional y relaciones internacionales de Vitoria-Gasteiz. №. I, 20 I 2, págs. 235-308.

PÉREZ DE ARMIÑO, Karlos, "El concepto y el uso de la seguridad humana: análisis crítico de sus potencialidades y riesgos", en Revista CIDOB d'afers internacionals, N. 76, 2006, pp. 59-77

PNUD, Human Development Report 1994, New York, Oxford, Oxford University Press, 1994. 
PRESTIANNI, Security and migration. Economic interest and violation of basic rights. The cases of Libya, Niger and Egypt, ARCI, 2019. Disponible en: https:/www.arci.it/app/uploads/2019/05/report-2019-inglese-normal.pdf, última consulta el 02/12/2019

PUIG, Oriol, PÉREZ, Marta, Níger gendarme de Europa, Informe de Oxfam Intermón n 52, julio 2019

ROTBERG, Robert, "Failed States, Collapsed States, Weak States: Causes and Indicators" en When States Fail: Causes and Consequences, Princeton, NJ: Princeton University Press, 2004).

SANHAUJA, José, y SCHUNEMANN, Julia, "El nexo seguridad-desarrollo: entre la construcción de la paz y la securitización de la ayuda” en Sanhauja, J. (coord.): Construcción de la paz, seguridad y desarrollo. Visiones, políticas y actores, ICEI, Madrid, 2012.

SCHMIDT, Jessica, "Intuitively neoliberal? Towards a critical understanding of resilience governance" en European Journal of International Relations, Vol 2I, Issue 2, 2015.

SERVICIO EUROPEO DE ACCIÓN EXTERIOR (SEAE), Estrategia Global para la Política Exterior y de Seguridad de la Unión Europea, 2016.

SPEAR, Joanna,WILLIAMS, Paul, Security and development in global politics. A critical comparison, Georgetown University Press, Washington, D.C., 2012.

STERN, Maria, OJENDAL, Joakim, “Exploring the security-development nexus”, en ARMER, Ramses, OJENDAL, J., SWAIN, Ashok, The Security-development Nexus: Peace, Conflict and Development, Athem Press, Londres, 2013.

VENTURI, Bernardo, The EU and the Sahel: A Laboratory of Experimentation for the Security-Migration-Development Nexus, Instituto Affari Internazionali, working paper 17/38, 2019, diciembre.

WAGNER,Wolfgan,ANHOLT, Rosanne, "Resilience as the EU Global Strategy's new leitmotif: pragmatic, problematic or promising?”, en Contemporary Security Policy, 37:3, 2016, 4I4-430 p. 416. 


\section{RELACIONES INTERNACIONALES}

Revista académica cuatrimestral de publicación electrónica Grupo de Estudios de Relaciones Internacionales (GERI)

Universidad Autónoma de Madrid, España

https://revistas.uam.es/relacionesinternacionales

ISSN 1699 - 3950

f facebook.com/RelacionesInternacionales

3. twitter.com/RRInternacional

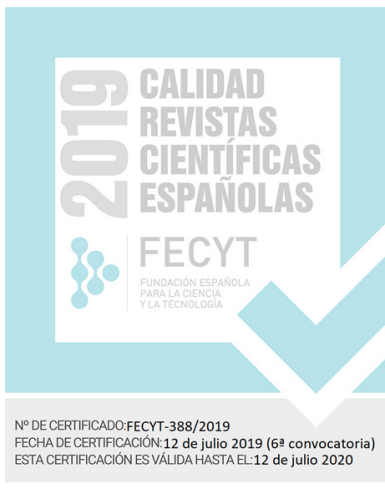

